\title{
IC TESTER USING PIC MICROCONTROLLER
}

\author{
S.Devika ${ }^{1}$, K.Anbarasan ${ }^{2}$, C.S.Chiranjeevi ${ }^{3}$, R.Haripriya ${ }^{4}$ \\ ${ }^{1}$ Department of Electronics and Communication Engineering, Jerusalem College of Engineering \\ ${ }^{2}$ Department of Electronics and Communication Engineering, Jerusalem College of Engineering \\ ${ }^{3}$ Department of Electronics and Communication Engineering, Jerusalem College of Engineering \\ ${ }^{4}$ Department of Electronics and Communication Engineering, Jerusalem College of Engineering
}

\begin{abstract}
The Integrated Circuit Tester is capable of testing both digital and analog ICs having 14 pin(74 series) and 8 pin(IC 555,LM741) respectively. It has flexibility in programming any number of ICs and can be tested with the memory constraints. The required input signal is applied to the IC for checking the condition of the IC and displays the output in the16x2 Liquid Crystal Display by displaying "GOOD" or "BAD" along with the IC number. The heart of the IC tester is PIC 16F877A Microcontroller which is programmed for deciding which IC, provide input, check the output and display the condition as mentioned.For digital ICs the possible input combinations are 00,01,10 and 11 and for analog IC input is given at VCC by checking the output and ground pins.
\end{abstract}

Keywords- PICmicrocontroller(16F877A), Embedded systems, IC Tester.

\section{INTRODUCTION}

In the electronics industry[1], with the drastic increase in the circuit complexity and requirement for higher levels of reliability, a major contribution involved is the cost involved for testing. For testing of IC, we require different hardware circuits for different IC's. We need a particular kind tester for testing a logic gate and another for testing the flip flops or shift registers. It increases the complexity of the circuit and consumes more time for the execution.

The purpose of the IC tester is it can easily check the IC within a short span of time. If there is any discrepancy in the result then it determines the gates which are good ones and which are bad ones. The manual operation or a human intervention includes testing each individual IC by making necessary connections and verifying the outputs for each gate by the truth table is a time consuming and a tedious process.

The IC which is to be tested is placed on the ZIF socket. The inputs are fed to the IC and primarily processed through the slave controlled board. The program is written in a manner that comparisons are to be made between the expected data from the truth table and the data that is obtained from the data bus. At last, in the LCD display the condition of the IC is displayed.

\section{LITERATURE SURVEY}

The Digital IC tester [2] is implemented in order to test the digital ICs to verify the faulty gates and the acceptable gates. The necessary input to the gates of the IC to be tested [3] which is placed in the ZIF socket is received from the microcontroller IC and the corresponding outputs are accumulated and sent to the same controller IC where the output is compared with the functional or logic table and if any discrepancy results, it displays the fault in the LCD display screen as well as in the PC screen.

With the implementation of the microcontroller units interfaced with PC makes the job much easier to receive data for the respective gates and process output and then display the result[4]. The operation of a PC based[5]controller is determined primarily by its program. The main advantage of this circuit is that whenever a new IC is to be tested and it does not include addition of hardware but a slight updation of the software is sufficient.

\section{PROPOSED SYSTEM}

This IC tester is affordable and user friendly which is constructed using PIC 16F877A microcontroller along with the display unit. Since it is programmable wide range of ICs can be tested which includes simple logic gates of 74 series and linear circuits such as LM741, IC555 and NE565 etc. Finally the display will shown in the LCD specifying whether the IC is good or bad and it is portable and easy to use.

\subsection{Hardware}

The various components of the IC tester is discussed as below

\subsubsection{Display Unit}

To display the result and for interaction with the user an HD44780 Liquid Crystal Display is used. This is a 2 line LCD with 16 input pins. The connection of these pins with the microcontroller block is shown in figure 1 . 


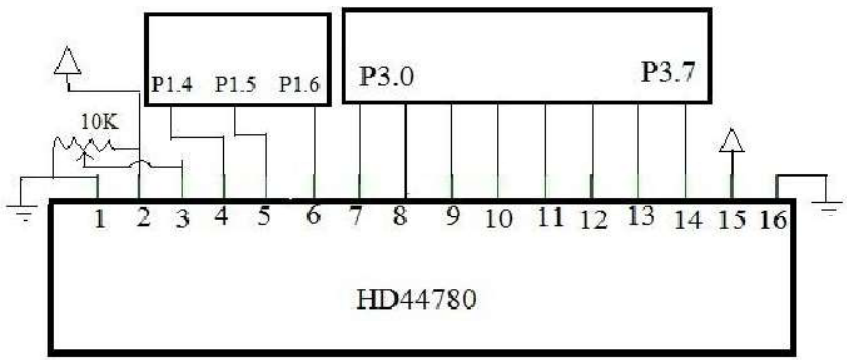

Pin Specifications of HD44780 LCD:

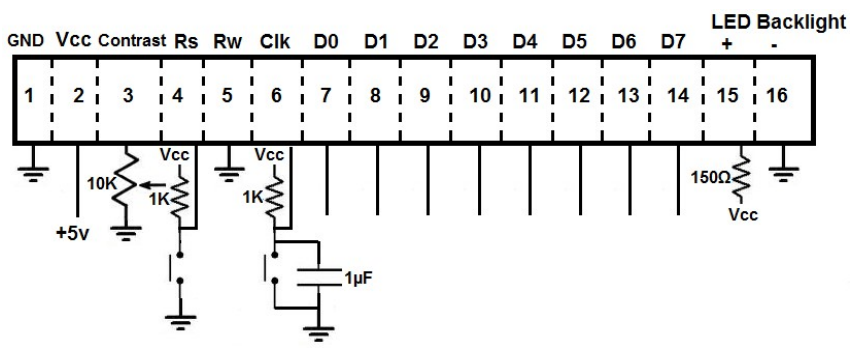

Fig 1: Display Unit Layout

\begin{tabular}{|c|c|c|}
\hline $\begin{array}{l}\text { PIN } \\
\text { NO }\end{array}$ & Symbol & Function \\
\hline 1 & VSS & GND \\
\hline 2 & VDD & $+5 \mathrm{~V}$ \\
\hline 3 & V0 & Contrast adjustment \\
\hline 4 & $\mathrm{RS}$ & H/L Register select signal \\
\hline 5 & $\mathrm{R} / \mathrm{W}$ & H/L Read/Write signal \\
\hline 6 & $\mathrm{E}$ & H/L Enable signal \\
\hline 7 & DB0 & H/L Data bus line \\
\hline 8 & DB1 & H/L Data bus line \\
\hline 9 & DB2 & H/L Data bus line \\
\hline 10 & DB3 & H/L Data bus line \\
\hline 11 & DB4 & H/L Data bus line \\
\hline 12 & DB5 & $\mathrm{H} / \mathrm{L}$ Data bus line \\
\hline 13 & DB6 & H/L Data bus line \\
\hline 14 & DB7 & H/L Data bus line \\
\hline 15 & A & $+4.2 \mathrm{~V}$ for $\mathrm{LED}$ \\
\hline 16 & $\mathrm{~K}$ & Power supply for BKL $(0 \mathrm{~V})$ \\
\hline
\end{tabular}

Fig 2.Funtions of the LCD pin

\subsubsection{Microcontroller Unit PDIP}

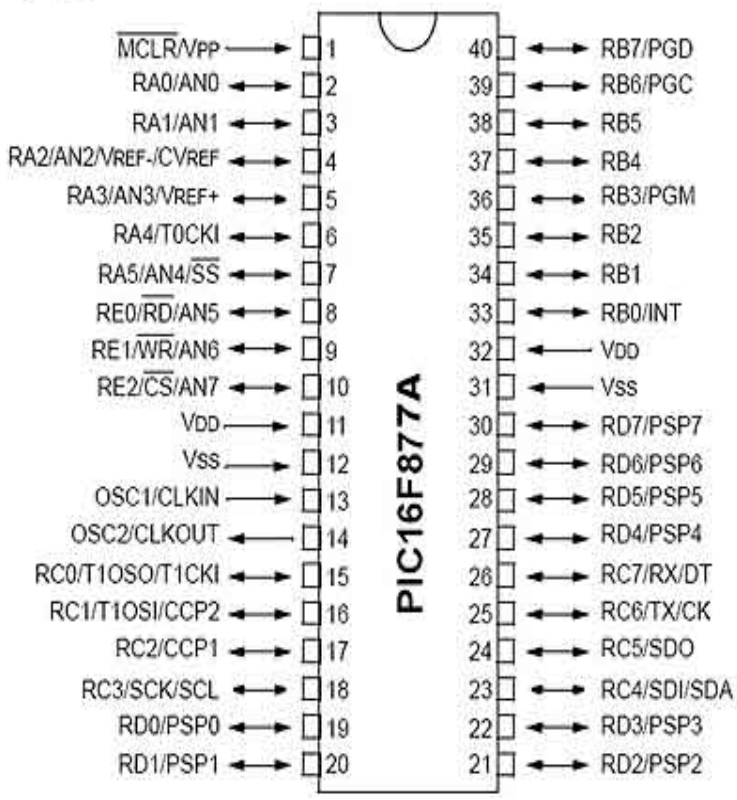

Fig.3: Pin diagram of Pic16f877a

For implementing this device PIC 16f877a microcontroller is used. The connections of different pins of the microcontroller with the peripherals allows the controller to check both the analog and digital ICs. The first 32 bytes of the register space are allocated to special purpose registers, the remaining 96 bytes are used for general purpose RAM.

PORT A -provides input to the input pin of the IC and directs the corresponding output to the display. PORT B have an interrupt-on-change feature. PORT C-determines the functions of its pins. PORT D -a 8 bit parallel slave port. PORT E- used as control signals .Timer modules are also available.

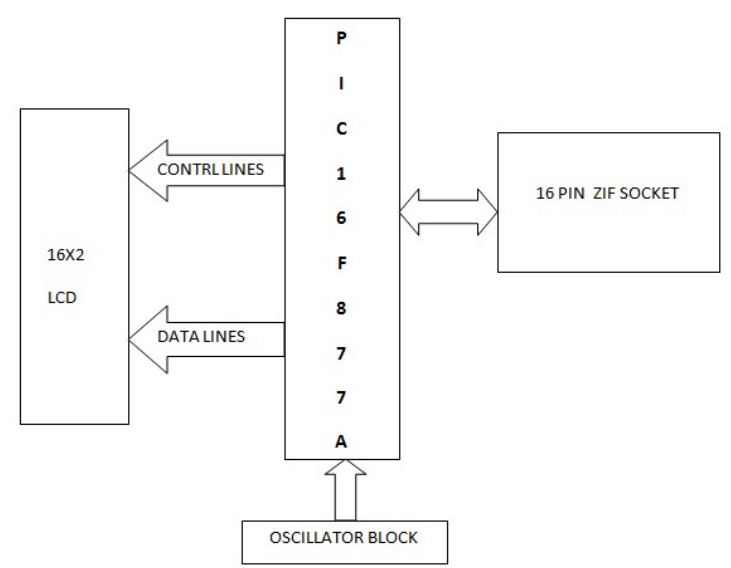

Fig.4 : Block diagram of ICTester

\subsection{Software}

The microcontroller executes the program loaded in its Flash memory. The basic simple $\mathrm{C}$ program is used to implement the tester operation. The concept of switch case conditional statement loops are used to find the IC number 
on the list. Once the IC number on the list was found then the input given to the IC of $5 \mathrm{v}$ is used to produce the output by verifying the corresponding matched sequence of properties of respective IC. Since the programming is so flexible the number of components of IC to be tested can be increased. As this controller is a EEPROM(Electrically Erasable Programmable Read Only Memory ) type offlash memory any ambiguity can be overcomed by simple modification in the program written in the controller.

\subsection{Flow Chart}

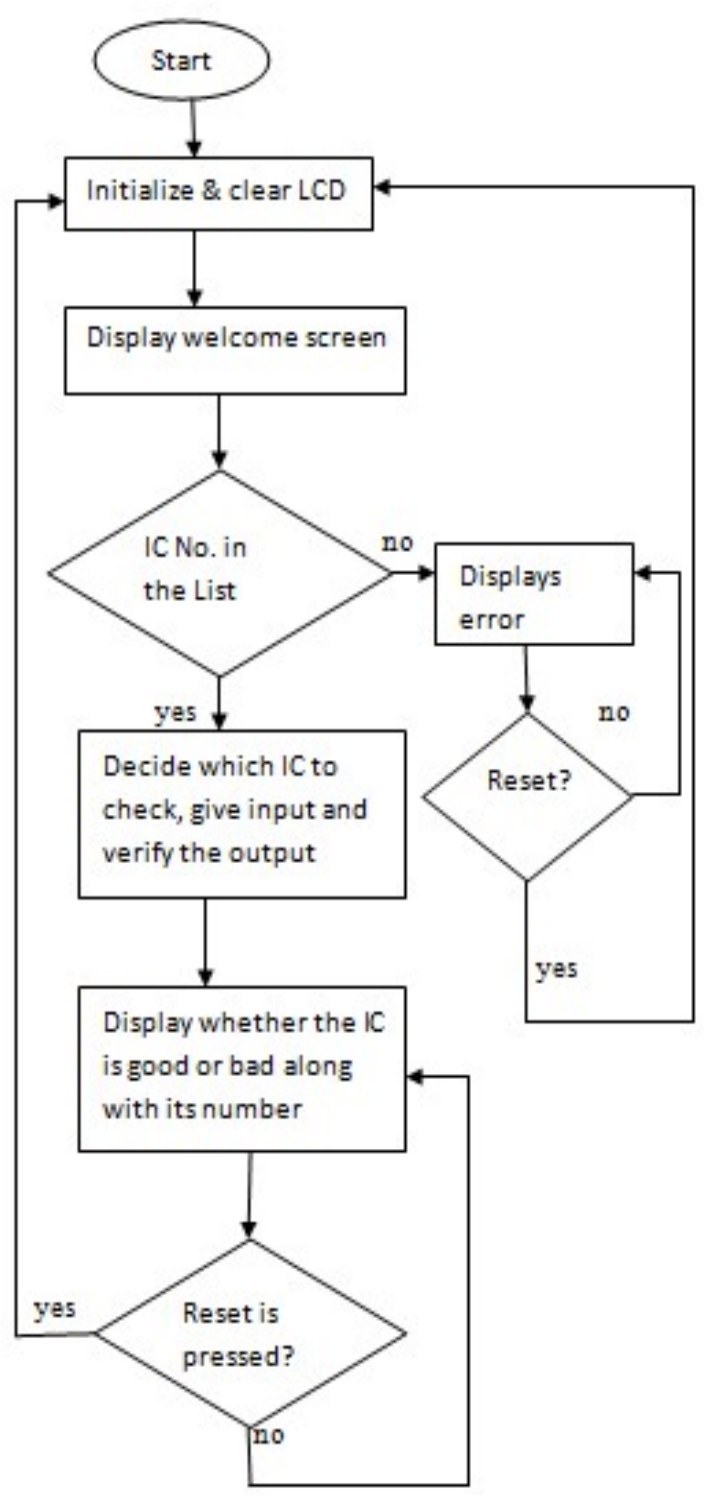

Fig 5. Flow chart

The description of the flow chart follows the procedure that after the switching ON of the power supply, LCD display screen will initialize and make the screen clear then displaying the welcome screen.After that when the IC is placed in the socket the controller checks for the IC no. in the list and provide proper input to the IC, checks and verifies the result and finally display the condition whether good or bad.

\section{Technical Specification}

\begin{tabular}{|l|l|}
\hline FAMILY & TTL,CMOS,PIC \\
\hline RANGE & Logic gates ,Ic555,Lm741 \\
\hline TEST SOCKETS & Two 16 pin ZIF socket for IC testing \\
\hline PACKAGE & DIP 14,16,20 and 24 pins \\
\hline DISPLAY & $16 X 2$ LCD DISPLAY \\
\hline ELECTRICAL & $230 \mathrm{~V}(+/-10 \%), 1$ phase,50Hz $(+/-2 \%)$ \\
\hline
\end{tabular}

\section{WORKING DESCRIPTION}

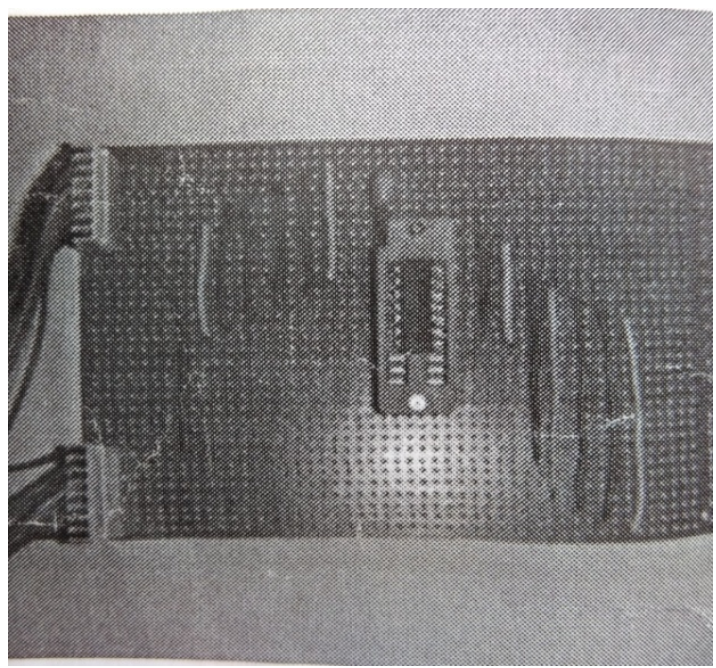

Fig 6 . PCB board

Working of the device can be described as, when the power supply is switched on, the power LED will glow. The IC to be tested is placed in the ZIF socket and the IC is locked by the lever. Once the IC is locked, the LCD display will show the condition of the IC by displaying whether it is Good or Bad along the IC number. In case the IC is not placed properly then it will display Error. The controller program is based on the switch case concept, so that when the particular IC matches the PIN number then the corresponding loop will be executed. For Digital IC the tester provides 0's and 1 's as inputs and verifies with the Truth table for the respective IC and displays the condition and for analog it checks the input supply VCC, Ground and Output pins.

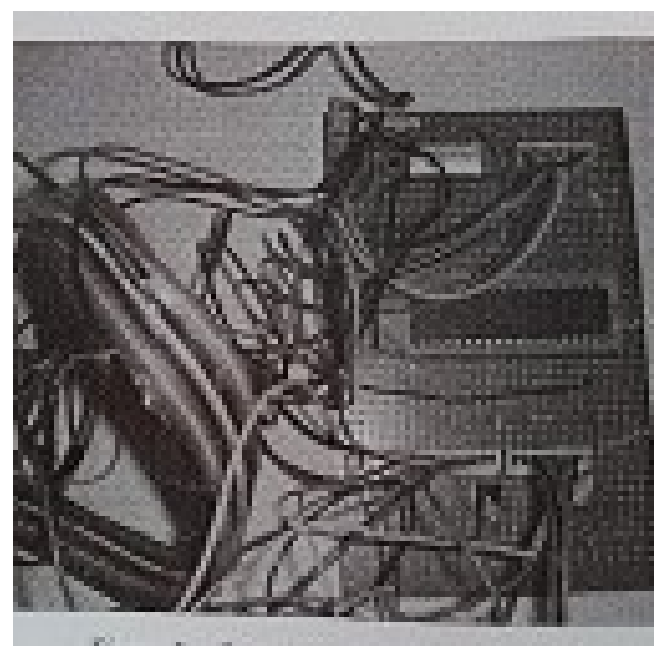

Fig.7 Snapshot of Microcontroller board 


\section{RESULT/EXPERIMENTAL OBSERVATIONS}

- When the supply is switched on the LCD screen will display the WELCOME text in the screen as shown in the Fig 8.

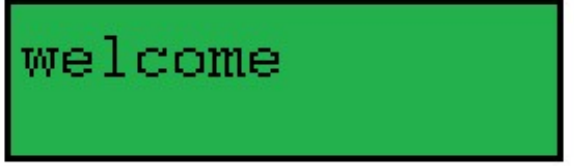

Fig.8 :LCD output at the start

- After placing the IC in the ZIF socket the processor generates the required input to the IC and find the number and also tells the condition.

For example the IC placed in the socket is an OR gate (7432), then the LCD displays the IC number 7432 and shows the IC condition whether good or bad. When the IC is in good condition the display is shown in the Fig.9

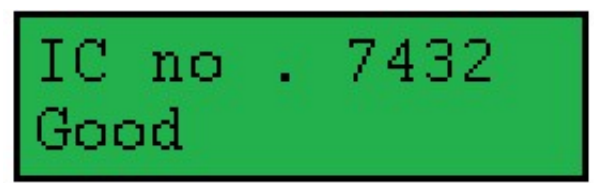

Fig 9 : IC in Good condition

- In case if the IC is not properly working then the display unit shows that the IC (7404) is Bad condition as shown in Fig 10.

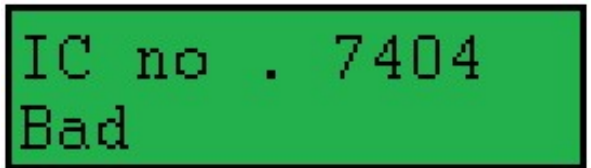

Fig 10. IC in Bad condition

- For an analog IC the output is similar to that of the digital IC ,example IC555 is tested by checking the supply pin , ground and the output pin. Finally displaying the condition as shown in fig 11

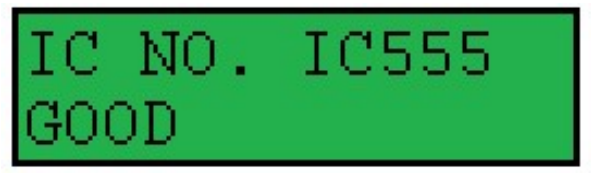

Fig 11. IC555

\section{CONCLUSION}

This Integrated circuit tester is programmed for both the digital and analog IC without the need of external keypad. However this device can be easily extended for the large number of ICs till the memory permits.

No rewiring is required .Only editing the source code accordingly will be needed. In case testing more number of pins two microcontrollers can also be used in master slave connection. One is for testing purpose and the other is for driving the display unit.

\section{REFERENCES}

[1].G.L West A microcontroller -controlled testing system for digital integrated circuits.

[2].GericA.Agawin, kristianM.M.FernandoclaudiusJose A.Yango,"PC based digital logic tester via USB port".

[3].Sherif Anus Mohammed,"In-Circuit testing for electronic board".

[4].A.Jutman, R.Ubar,"Laboratory training for teaching design and test of digital circuits."

[5].I.C.Rice, "Base your IC tester on a microprocessor'. 